\title{
Reporte de Vigilancia Tecnológica en Sistemas de Iluminación
}

\section{A Technological Surveillance Report about Lighting Systems}

\author{
Alexander Eduardo Florián-Villa a \& Rosa Elvira Correa ${ }^{b}$ \\ a Tecnólogo en mecánica automotriz, estudiante Institución Universitaria Pascual Bravo, (Semillero de investigación \\ SICAP), Medellín-Colombia, da.tobon@pascualbravo.edu.co \\ ${ }^{b}$ Doctor en ingeniería, docente Institución Universitaria Pascual Bravo, (Grupo de investigación GIIEN), \\ Medellín-Colombia, i.patinoar@pascualbravo.edu.co \\ a Magíster en automatización y control industrial, docente Institución Universitaria Pascual Bravo, (Grupo de \\ investigación GIIEN), Medellín-Colombia, karen.lemmel@pascualbravo.edu.co
}

Recibido: septiembre 30, 2019. Aceptado: diciembre 19, 2019

\begin{abstract}
Resumen
Las actividades antropogénicas, naturales y una era industrial altamente acelerada han conllevado a una gran concentración de gases de efecto invernadero en la atmósfera, incidiendo directamente en el proceso que se conoce como cambio climático. El sector encargado de la generación de energía está altamente involucrado en estos cambios, y es por esta razón que debe tomar cartas en el asunto, impulsando el desarrollo de tecnologías amigables que preserven el medio ambiente, diseñando dispositivos más eficientes, tanto en la generación como en la transmisión y el consumo final, de tal manera que se pueda garantizar un sostenibilidad económica y ambiental. En este documento se hace una exploración de las tecnologías utilizadas para generar la iluminación artificial utilizada en diferentes tipos de ambientes, y se realiza una clasificación de los sistemas de iluminación basada en la tecnología de la fabricación de la fuente emisora de luz; además, se recopilan datos de consumo energético y ciclo de vida en algunas luminarias. Desde el punto de vista normativo, se hace una recopilación de información acerca de la legislación colombiana para el sector de iluminación, listando algunas leyes y decretos dispuestos por para su obligatorio cumplimiento, las cuales propenden por el cuidado de la vida humana, vegetal y animal, y en general de los equipos y dispositivos utilizados en los sistemas de energía.
\end{abstract}

Palabras clave: Iluminación artificial, luminaria, bombilla, eficiencia energética, RETILAP.

\begin{abstract}
Anthropogenic, natural activities, and a highly accelerated industrial era have involved a large concentration of greenhouse gases in the atmosphere, directly affecting the climatic change process. The energy generation sector is highly involved in these changes, so they must take responsibility for the matter, supporting the development of new technologies to protect the environment, designing efficient devices for generation, transmission, and final customer use in order to guarantee economic and environmental sustainability. This paper makes an exploration of the technologies used for the artificial illumination of different kinds of environments, classifying the lights according to the manufacturing technology of the emitting source. Besides, the work involves a compilation of data about energy consumption and life cycle in some luminaires. For the regulatory aspect, this work also compiles information about Colombian legislation for the lighting sector, listing some laws and decrees provided by law for mandatory compliance, which tend to care for human life, plant, animate, and the equipment and devices used in energy systems.
\end{abstract}

Keywords: Artificial lighting, luminaire, bulb, energy efficiency, RETILAP.

\section{INTRODUCCIÓN}

El desarrollo tecnológico ha jalonado el avance social de la humanidad desde la Revolución Industrial hasta la actualidad, impulsado por los sistemas eléctricos que sostienen el crecimiento económico a partir de la generación de energía [1], [2]. No obstante, se ha incrementado la preocupación mundial por los efectos que la expansión del mercado y la economía globalizada producen en el medio ambiente [3], [4]. El sector eléctrico no es ajeno a este fenómeno, y se considera en el segundo lugar entre los agentes contribuyentes al calentamiento global [5], a través de elementos como la generación térmica [6] y los sistemas de iluminación artificial; en estos últimos, en particular, se encuentra una creciente necesidad de desplazar paulatinamente los sistemas convencionales por sistemas de iluminación artificial que sean sostenibles y compatibles con el medio ambiente [7].

\footnotetext{
Citar como:

Alexander Eduardo Florián-Villa \& Rosa Elvira Correa. "Reporte de Vigilancia Tecnológica en Sistemas de Iluminación" Revista CINTEX, Vol. 24(2), pp. 35-45. 2019
} 
La iluminación fue el primer beneficio ofrecido por los servicios eléctricos y aún continúa siendo uno de los usos finales eléctricos más grandes [8]. La primera fuente de luz eléctrica que se comercializó fue la lámpara incandescente a fines del siglo XVIII. Aunque la lámpara incandescente cambió muy poco en los siguientes cien años, en términos de rendimiento, se introdujeron nuevas fuentes de iluminación que entregan más luz por menos cantidad de energía durante ese período de tiempo, la mayoría de las cuales mejoraron en su rendimiento para convertirse en el producto utilizado en la actualidad [9]. Según las estimaciones de la Agencia Internacional de Energía (AIE), alrededor del 19\% de la electricidad utilizada en el mundo en 2015 se usaba en iluminación [10]. La demanda de iluminación representa casi un quinto del consumo total de electricidad en un hogar promedio; se espera que la energía utilizada para la iluminación continúe con una tendencia al alza; en particular, la demanda de iluminación surge de una combinación de efectos que incluyen la disponibilidad de iluminación natural y las características de los ocupantes. La mayoría de las tecnologías de iluminación actuales se pueden mejorar enormemente y, por lo tanto, las cargas de iluminación presentan un enorme potencial de ahorro de electricidad [10].

Sin embargo, al igual que con las emisiones de gases de efecto invernadero, la iluminación artificial puede llegar a considerarse como un contaminante ambiental globalmente extendido. Se estima que el $23 \%$ de la superficie terrestre [11] está expuesta al resplandor artificial del cielo (luz artificial que se dispersa en la atmósfera y se refleja de nuevo en el suelo). El grado de exposición a la iluminación aumentó en todos los ecosistemas terrestres mundiales entre 2008 y 2012, y los que son importantes para la conservación de la biodiversidad a menudo son los más afectados [12]. La exposición a la luz artificial no se limita a los ambientes terrestres, pues se han reportado estimaciones que indican que el $22 \%$ de las regiones costeras del mundo [13] están experimentando algún grado de exposición a la iluminación artificial. La cantidad de luz artificial también está aumentando en las áreas terrestres protegidas en Europa, Asia y América del Sur y Central [14], y casi el 15\% de las áreas marinas protegidas del mundo [15]. Dado que se proyecta que más del $95 \%$ del aumento de la población mundial ocurrirá en las ciudades de los países económicamente en desarrollo durante los próximos 50 años [16], y los niveles de contaminación lumínica están estrechamente asociados con la densidad de población y la actividad económica [17], se espera que la iluminación artificial continúe expandiéndose tanto en extensión como en intensidad espacial durante el siglo XXI.

Todos estos factores, junto con las preocupaciones asociadas con la eficiencia energética y el ahorro de costos asociado, están impulsando la investigación y el desarrollo de las tecnologías de iluminación. Actualmente, se está produciendo un cambio de las tecnologías de iluminación convencionales (incandescente, fluorescente, descarga de alta intensidad) a la iluminación LED. La iluminación LED ahora es más eficaz que cualquiera de las tecnologías de iluminación convencionales, y aun cuenta con margen para mejorar aún más [18]. Este trabajo presenta los resultados de una vigilancia tecnológica en sistemas e iluminación, describiendo inicialmente la cantidad de artículos y patentes en bases de datos, para luego presentar una breve clasificación de los sistemas de iluminación de acuerdo con su tecnología. Más adelante, se presentan algunas cifras relacionadas con el sector en Colombia, así como el marco regulatorio que rige al sistema de iluminación y alumbrado público en el mismo país.

En el segundo, se determina la carga máxima que podría soportar la estructura sin sufrir deformaciones permanentes; en el tercer caso, se obtiene la carga máxima que podría soportar la estructura sin sufrir una falla localizada.

\section{RESULTADOS DE LA BÚSQUEDA DE INFORMACIÓN EN BASES DE DATOS DE PUBLICACIONES CIENTÍFICAS Y PATENTES}

Esta sección presenta una investigación documental, donde se analizaron diferentes publicaciones científicas sobre las tendencias en la iluminación a nivel mundial, bajo el modelo de vigilancia tecnológica. La vigilancia tecnológica, como estrategia y parte del sistema del proceso de inteligencia competitiva, es una herramienta de captación de información relevante, en un tema específico y determinado, en el cual se quiere tener un conocimiento suficientemente amplio para la toma estratégica de decisiones basadas en el conocimiento del entorno, el producto, la competencia, los avances tecnológicos, entre otros [19].

Como resultado de esta vigilancia, se pretendía resaltar la actualidad de los sistemas de iluminación, y la evolución que estos han tenido a través del tiempo, elementos que serán descritos en secciones subsiguientes. De acuerdo a los resultados de la búsqueda de información científica y de patentes, los sistemas de iluminación están siendo un gran tema de investigación, desarrollo y comercialización a nivel mundial y regional, la eficiencia, las diversas aplicaciones y la preocupación por el desarrollo de tecnologías limpias han estado impulsando a que la iluminación sea un tema de total actualidad en el mundo científico y comercial, dando como resultado que

Revista Cintex | Vol24(2) | julio-diciembre | 2019 
muchos países estén haciendo leyes para disminuir el impacto ambiental causado en toda la cadena de iluminación artificial.

\subsection{Entorno: Artículos Académicos}

Las figuras 1 y 2 presentan el número de publicaciones científicas por año que se ocupan del tema de sistemas de iluminación; esta información se han extraído de bases de datos de alto impacto y que cobijan varias revistas científicas que publican artículos en este tema, estas gráficas muestran la constante tendencia al alza de las investigaciones realizadas en sistemas de iluminación, lo que deja como conclusión que este es un tema de interés y de alto impacto para la comunidad científica, los gobiernos y la sociedad en general. Los países que más investigaciones publican a nivel mundial en el tema de sistemas de iluminación en este momento son, en orden de mayor número de publicaciones: Estados Unidos de América, Japón, Alemania, China, Francia, Reino Unido, e India.

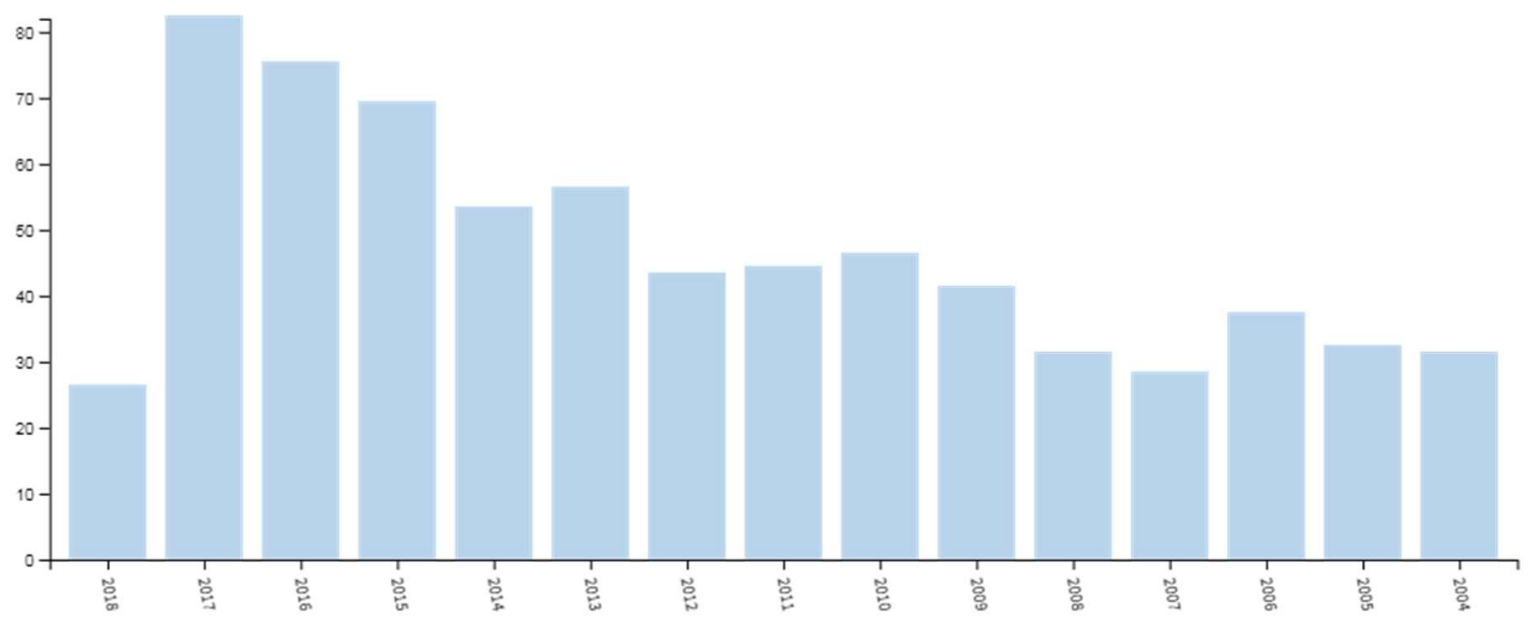

Figura 1. Número de publicaciones por año registradas en la base de datos Web of Science, para la temática "lighting"

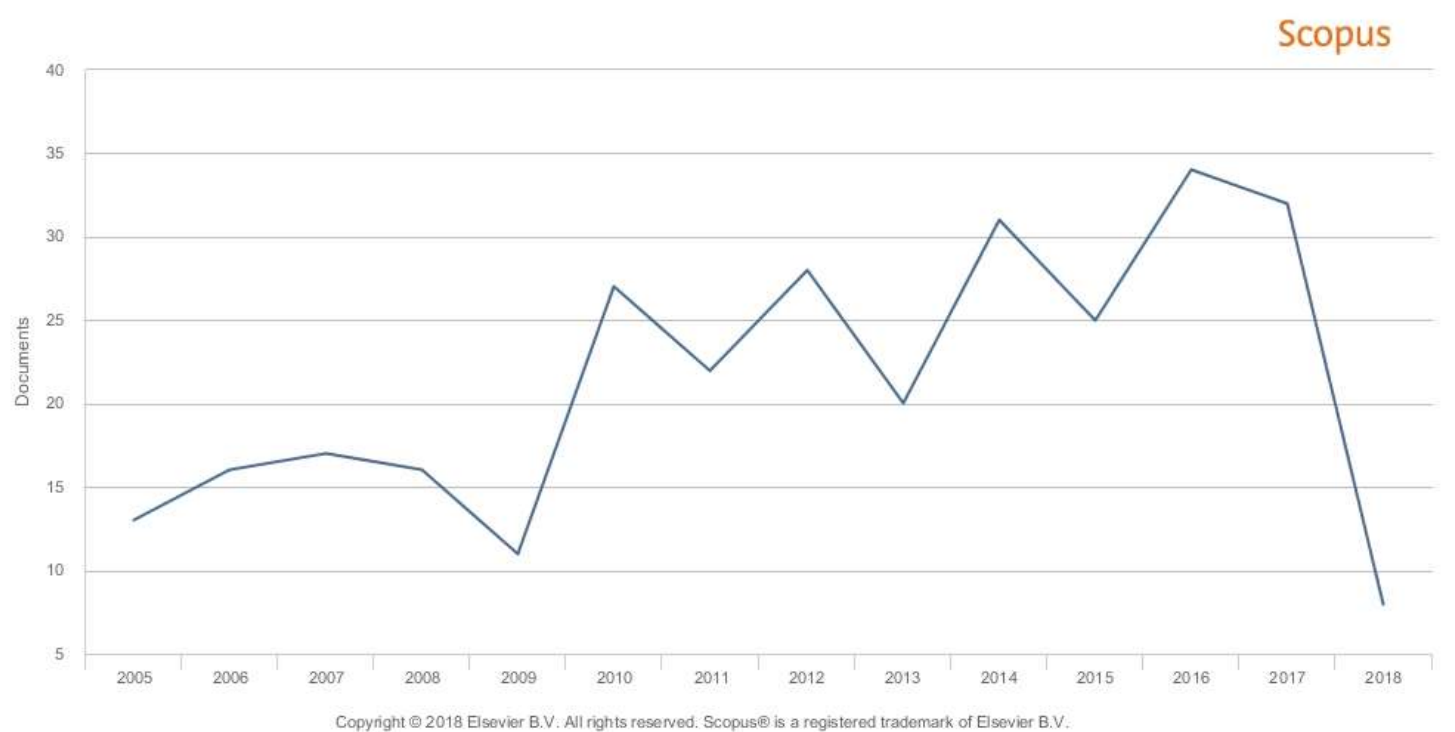

Figura 2. Número de publicaciones por año registradas en la base de datos Scopus para la temática "lighting"

\subsection{Entorno: Patentes}

Actualmente hay 22.262 patentes estadounidenses otorgadas en relación con las tecnologías de LED, la mayoría de las cuales fueron presentadas en los últimos 5 a 10 años. Las 15 principales organizaciones propietarias de patentes representan más del $25 \%$ de todas las patentes concedidas para la tecnología y las aplicaciones LED [20]. 
Según el World Intellectual Property Office (WIPO), el número de aplicaciones en patentes relacionada con sistemas de iluminación, creación de celdas luminosas, y demás componentes asociados a la generación de luz artificial se encuentra en una pendiente ascendente, debido a la diversidad de aplicaciones, a los nuevos materiales, y a la gran necesidad de ahorrar energía, es por esto por lo que algunos países han estado investigando y desarrollando tecnología en este campo.

\section{IDENTIFICACIÓN DE LAS PRINCIPALES TECNOLOGÍAS EN LA CONSTRUCCIÓN DE LUMINARIAS}

La iluminación es una de las necesidades básicas en la sociedad moderna. En ambientes al aire libre; como caminos, calles, aparcamientos, y parques; la iluminación permite la detección de personas, vehículos y otros objetos cuando oscurece. La iluminación contribuye a la sensación de seguridad y comodidad de los usuarios. Es por esto por lo que los sistemas de iluminación juegan un papel primordial en la sensación de seguridad de las personas; los sistemas de iluminación son el conjunto de elementos que proporcionan en primera instancia visibilidad, y en segunda instancia agregan valor estético a los ambientes iluminados de una manera óptima en la distribución de energía y en el consumo energético.

Existe una gran variedad de luminarias, y también existen diversas formas de hacer clasificación de estas, entre las que se pueden encontrar las siguientes:

- Según la tecnología de fabricación.

- Según la forma de dispersión de la luz.

- Según el grado de protección.

- Según su grado de seguridad eléctrica.

- Según su aplicación.

A continuación, se presentará un análisis de las luminarias clasificadas por su tecnología de fabricación y elementos constitutivos, debido a que los últimos avances en la tecnología de iluminación están haciendo las luminarias cada vez más eficientes, amigables con el medio ambiente y con un costo económico cada vez más bajo.

\subsection{Lámparas incandescentes}

Este tipo de luminaria se caracteriza porque la producción de luz de debe al calentamiento de un filamento por el cual circula corriente eléctrica, la cual hace que se genere la luz. La construcción básica de las luminarias incandescentes está dada por una lámpara que se encuentra al vacío, o que en su interior se encuentra algún tipo de gas noble (nitrógeno - argón). Una variación de las lámparas incandescentes son las lámparas alógenas en las cuales el filamento está dentro de una burbuja de cuarzo, dentro de la cual se encuentra un gas [21].

Las lámparas incandescentes son altamente ineficientes en todas las categorías de evaluación del ciclo de vida, entre las cuales se encuentra su alto consumo energético, producción de calor y grado de contaminación posterior al uso. Sin embargo, en ciertas aplicaciones especiales de iluminación de alta intensidad, como la iluminación en estudios cinematográficos y la proyección de películas cinematográficas, es aun masivamente utilizada esta tecnología. En la actualidad, estas lámparas están prohibidas en gran parte del mundo y su desarrollo se encuentra detenido debido a la aparición de tecnologías más eficientes y amigables con el medio ambiente [22].

\subsection{Lámparas de alta intensidad de descarga.}

Las luminarias de alta intensidad tienen electrodos los cuales se energizan para hacer circular la corriente eléctrica a través de ellos; estos electrodos están contenidos dentro de un tubo para la generación del arco eléctrico, el cual excita los gases contenidos en su interior (vapor de mercurio y polvos fluorescentes) para así generar la luz en el tubo de descarga [23].

Las lámparas de descarga de alta intensidad (HID) son aceptadas en todo el mundo como una de las principales fuentes de luz artificial para iluminación vial, e iluminación deportiva, y recientemente, la lámpara de descarga de alta intensidad (HID) se ha vuelto cada vez más popular debido a sus características de alta eficacia luminosa, larga vida útil y buena reproducción del color.

Durante los últimos 15 años, una serie de nuevas aplicaciones para lámparas $\mathrm{MH}$ han sido posibles gracias al desarrollo de lámparas compactas de baja potencia (32-150 W), Un número de grandes empresas de iluminación han estado invirtiendo fuertemente en el desarrollo de pequeñas lámparas compactas HID para aplicaciones tales 
como sistemas de luces para vehículos, debido a su alta eficiencia de radiación y su prometedor potencial de mercado [24].

\subsection{Lámparas a base de LED}

Los diodos emisores de luz (LED, Light Emitting Diode), son dispositivos ampliamente utilizados en los últimos años en los sistemas de iluminación; los diodos emisores de luz disipan la energía en un haz de luz, la cual en muchos casos es proporcional a la cantidad de corriente eléctrica aplicada al dispositivo. La figura 3 describe los elementos de una luminaria LED típica.

Los LED tienen distintas aplicaciones, aunque anteriormente su implementación estaba reducida únicamente a indicadores de tensión y corriente en circuitos electrónicos, debido a su baja potencia y efectividad; estos emitían luz rojiza de baja intensidad [10]. Sin embargo, su desarrollo evolucionó hasta formar parte de complejos sistemas de iluminación; en la actualidad las luces LED tienen diferentes aplicaciones en diversos escenarios, desde sistemas de iluminación vial, pasando por grandes y avanzados avisos publicitarios hasta ser la última tecnología para televisión inteligente [25].

La expansión de las luminarias LED se intensificó mediante el espectacular desarrollo de las tecnologías para iluminación de estado sólido, estimulado por la directiva de la Comisión Europea que prohibió el uso ineficaz de las lámparas incandescentes en 2016 para reducir el consumo de energía y aumentar la independencia energética [25]. Al reemplazar la mayoría de las lámparas incandescentes en 2030, se estima que la lámpara LED capturará hasta un $74 \%$ de la cuota de mercado, reduciendo el consumo de energía en un $46 \%$ por un ahorro de energía de 30 mil millones de dólares en comparación con el precio de la energía en 2012 en EE. UU [26]. La creciente cuota de mercado de la lámpara LED se puede atribuir al aumento de la eficacia de la luminaria, la mayor integración de LED con una variedad de aparatos eléctricos y la reducción gradual del costo inicial de adquisición de LED [26].

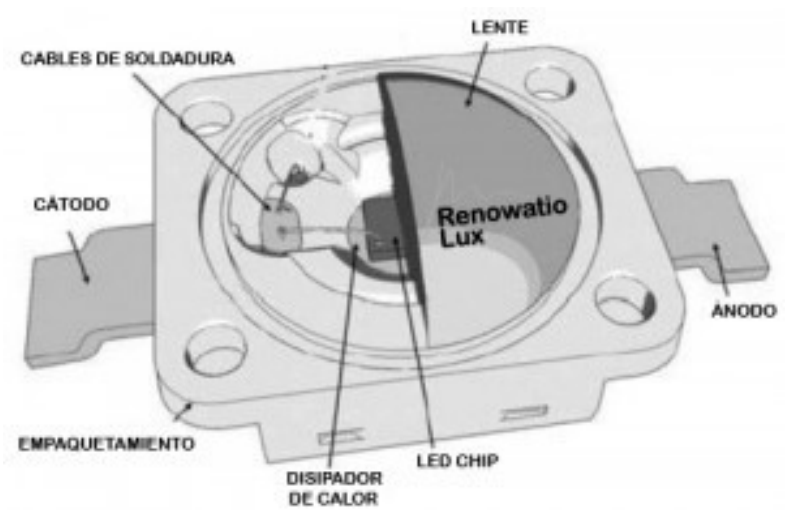

Figura 3. Elementos de una luminaria LED típica (Adaptado de [27]).

\subsection{Lámparas fluorescentes.}

Son luminarias tubulares en cuyo interior se encuentra gas a baja presión, y que generan luz gracias a que en su interior unos electrodos energizados producen un arco eléctrico que vaporiza el mercurio que se encuentra dentro del tubo. Los electrodos de tungsteno con los que generalmente se fabrican las lámparas incandescentes tienen una gran similitud con los de las lámparas fluorescentes; estos se diseñan según la forma de arranque de la lámpara, los electrodos se ubican en las cabeceras de la lámpara para la producción del arco eléctrico que genera calor para hacer que los electrones del gas en el interior de la lámpara se ionicen y generen una emisión primaria de luz ultravioleta, la cual no es visible por el ojo humano, pero que puede excitar el material fluorescente que recubre las paredes interiores de la lámpara, y que transforma esta iluminación ultravioleta en un haz de luz visible por el ojo humano. Este tipo de luminaria se puede encontrar en diferentes configuraciones geométricas, las cuales se usan según la necesidad específica [28].

Las lámparas fluorescentes aparecieron en el mercado en la década de 1980 como una estrategia para disminuir el calentamiento global, y combatir los gases de efecto invernadero provocados por el uso de energía eléctrica, ya que este tipo de lámpara presentaba un menor consumo energético que su competencia directa 
(lámparas incandescentes), lo cual las hizo altamente populares y ampliamente utilizadas tanto en la industria como en los hogares, e hizo de este tipo de lámpara un ícono de conservación del planeta.

En la actualidad existen serias preocupaciones sobre los verdaderos beneficios de las lámparas fluorescentes, las cuales podrían ser peores para el medioambiente, debido a su contenido de mercurio, el impacto de los tiempos de encendido cortos en la vida útil de las lámparas, la energía utilizada durante su complejo proceso de fabricación y disposición final; debido a que este tipo de luminaria está fabricado con una gran cantidad de elementos nocivos para la salud y el ambiente, su disposición final no es fácil de realizar, de hecho algunas organizaciones catalogan esta luminaria como objeto peligroso a la hora de ser reciclado [29].

\subsection{Lámparas de inducción.}

Las lámparas sin electrodos son una fuente de iluminación de cuarta generación, junto con los LED. Las lámparas de inducción son básicamente una variación de las lámparas fluorescentes, con la diferencia de que las lámparas de inducción no tienen electrodos para generar arco eléctrico, y que el encendido y el apagado es de manera instantánea. Las lámparas de inducción son altamente utilizadas debido a su bajo consumo energético y a sus bajos costos de mantenimiento, una característica que no juega a su favor es su alto costo inicial a la hora de adquirir este tipo de luminaria.

En estas lámparas la luz se genera por medio de la descarga de un gas y una inducción que alimenta a un generador electrónico de alta frecuencia; la corriente eléctrica inducida hace que las partículas de gas metálico se aceleren y colisionen para así generar una radiación ultravioleta la cual interactúa con las capas fluorescentes de la lámpara, interacción que hace que se cree el haz de luz visible [30].

\section{PANORAMA ACTUAL EN EL SECTOR DE LA ILUMINACIÓN}

La energía es un bien fundamental para el desarrollo de todas las actividades comerciales e industriales, así como para el bienestar de los hogares. Actualmente, la administración de energía para la iluminación exterior se ha incrementado en varias ciudades, y dado que las lámparas de sodio de alta presión (HPS) han sido un estándar en iluminación, por sus buenas características de difusión de luz y la carencia de otras tecnologías para cumplir con los requisitos mínimos necesarios para esta aplicación. Las luminarias HPS han sido ampliamente utilizadas a pesar de su alto consumo energético; sin embargo, y en aras de ahorrar energía, el alumbrado público con diodos emisores de luz (LED) se ha convertido rápidamente en una nueva tendencia.

El uso mundial de electricidad para la iluminación fue de unos 2.000 TWh en 2013, lo que representó casi el $20 \%$ del uso global de electricidad en el mundo. Con el paso de los años, el uso general de la electricidad en el mundo ha crecido constantemente, lo que es una tendencia alarmante en lo que respecta a las emisiones de gases de efecto invernadero y al cambio climático; por ejemplo, aumentó hasta en un $20 \%$ entre 2000 y 2013 [18]. Teniendo en cuenta que alrededor del $75 \%$ de las instalaciones de iluminación interior en los países desarrollados son ahora obsoletas, la retroalimentación de iluminación es una de las alternativas más prometedoras que podría ralentizar significativamente la tendencia del uso de electricidad en el mundo. Algunas Investigaciones han demostrado que, para espacios interiores, se puede lograr un considerable ahorro de energía mediante el uso de sistemas de control de iluminación (LCS) [31].

De acuerdo con la revisión del Balance Energético Colombiano [27], en el año 2015 el país consumió 207.370 TJ en energía eléctrica; esta cantidad de energía consumida se distribuye en: iluminación (31\%), aire acondicionado (22,8\%) y refrigeración (13,9\%). La figura 3 presenta el consumo de electricidad en Colombia según su uso; esta imagen demuestra lo representativo que es el consumo de energía eléctrica usada en iluminación, razón por la cual es indispensable buscar alternativas que permitan la disminución y optimización de la energía usada en esta aplicación. 


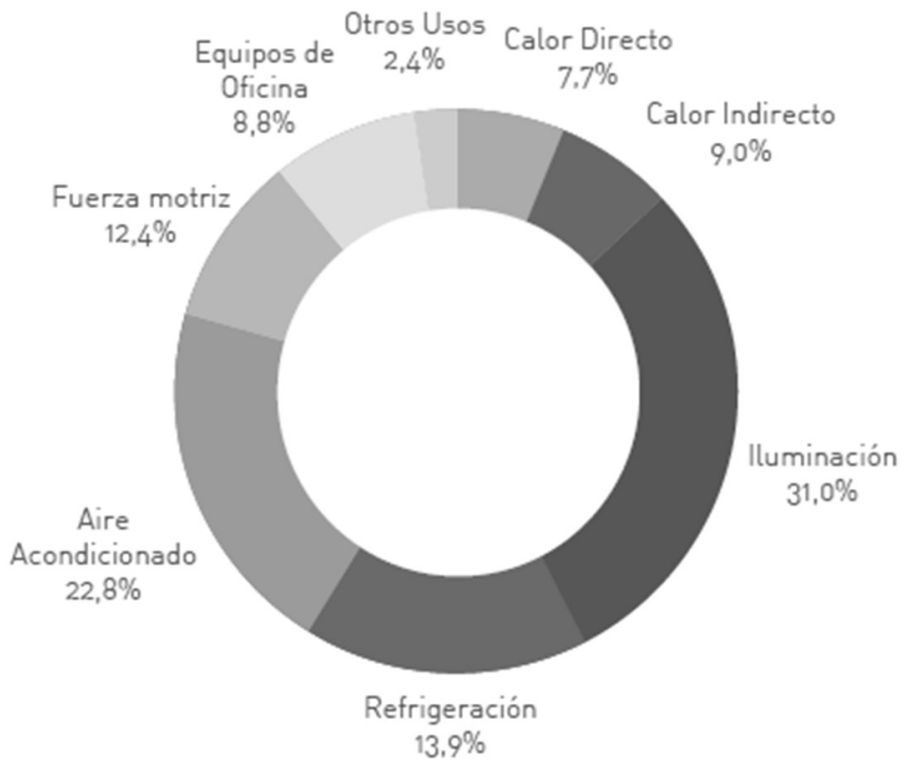

Figura 3. Consumo de electricidad en Colombia según su uso (Adaptado de [27]).

Pasando al ámbito internacional, actualmente los ahorros de energía en EE. UU., provenientes de la iluminación LED se estiman en 0.3 Quads (cuatrillones de unidades térmicas británicas), equivalentes a aproximadamente $30 \mathrm{TWh} / a n ̃ o$. Se pueden esperar ahorros similares de la penetración de los LED en Europa. Se espera que el consumo total de energía de iluminación aumente durante las próximas dos décadas. Sin embargo, para el año 2035, si se alcanzan los objetivos de costo y rendimiento del Departamento de Energía de EE. UU. La iluminación LED podría ahorrar 5.1 Quads de energía primaria por año, equivalente a 500 TWh/año, y que representan posiblemente el $5 \%$ del presupuesto total de energía de los EE. UU. En todo el mundo, los ahorros serían 3 a 5 veces más altos [31].

\subsection{Tipos de tecnologías usadas en iluminación}

La iluminación eléctrica representa hoy en día alrededor de un quinto del uso de la electricidad en el mundo y se prevé alarmantemente que esta cifra aumentará en un $40 \%$ en los próximos 15 años [32]. Actualmente, en Colombia existe una tendencia a la modernización de los sistemas de iluminación empleando tecnologías de tubos fluorescentes tipo T5 y LED, dado a que como se mencionó anteriormente estas tecnologías son las que están liderando todo en entorno de ahorro de energía a nivel mundial; aunque persisten tecnologías como de tubos fluorescentes tipo T12 y T8 que se niegan a abandonar el mercado de iluminación en el corto plazo. En la figura 4 se ilustra el uso de los diferentes tipos de tecnologías de iluminación empleados en el sector terciario (Comercio, mantenimiento y reparación de vehículos automotores y motocicletas, sus partes, piezas y accesorios; comercio al por menor de combustibles y lubricantes para vehículos automotores, entre otras) en Colombia [33].

La figura 4 muestra que se está impulsando el uso de tecnologías eficientes para el ahorro de electricidad en sistemas de iluminación; este cambio de paradigma se genera gracias a los prometedores ahorros de las luminarias LED. El principal impulsor de este cambio ha sido la eficiencia energética y el ahorro de costos asociados a la misma. La iluminación LED ahora es más eficaz que cualquiera de las tecnologías de iluminación convencionales, y cuenta con margen para mejorar aún más. Los paquetes de LED convertidos a fósforo, a corto plazo tienen el potencial de mejorar la eficacia entre $160 \mathrm{Im} / \mathrm{W}$ (ahora) y $255 \mathrm{~lm} / \mathrm{W}$. A largo plazo, los paquetes de LED mezclados a color tienen el potencial para niveles de eficacia concebiblemente tan altos como $330 \mathrm{Im} / \mathrm{W}$, aunque alcanzar estos niveles de rendimiento requiere de avances en la eficiencia del LED verde y ámbar [18]. 


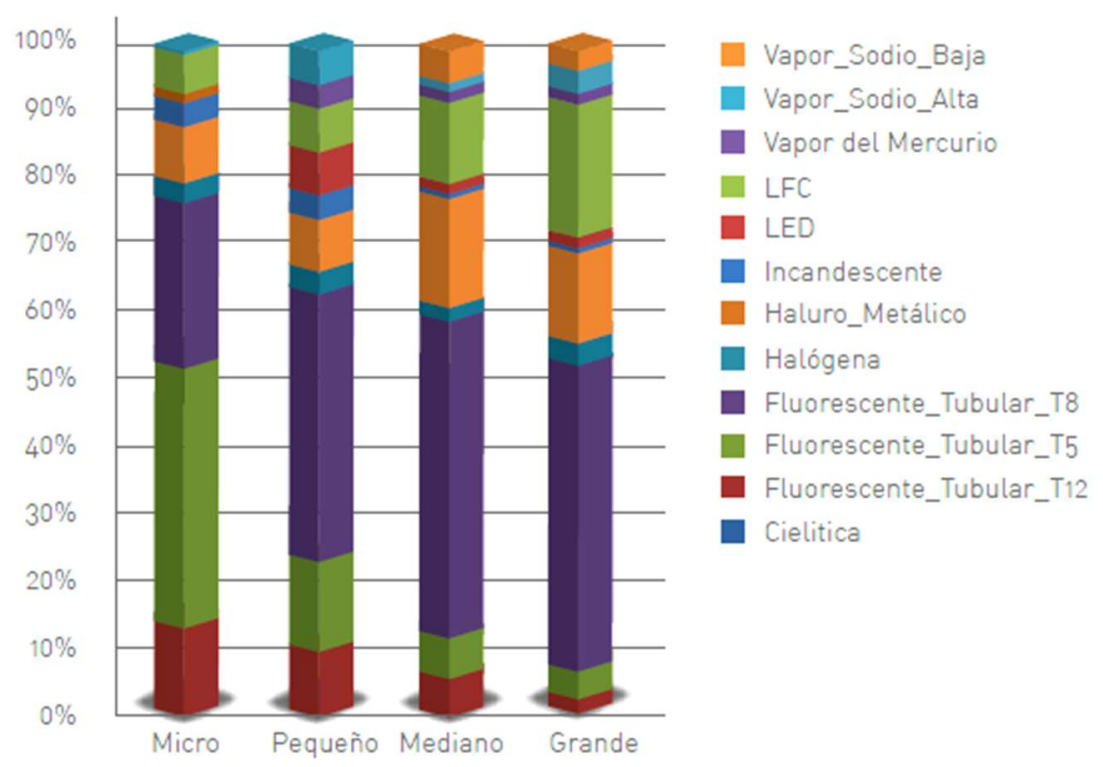

Figura 4. Tipos de tecnologías usadas en iluminación en Colombia ([33]

La iluminación led ha avanzado hasta el punto en que es la mejor opción para casi todas las aplicaciones de iluminación. En los últimos 15 años, la eficacia de los paquetes de LED blanco frío ha mejorado de alrededor de $25 \mathrm{~lm} / \mathrm{W}$ (lúmenes por vatio) a más de $160 \mathrm{~lm} / \mathrm{W}$, dependiendo de la calidad del color y las condiciones del disco. Simultáneamente, los costos de los paquetes de LED han disminuido hasta el punto en que los productos de iluminación LED pueden ser competitivos con los productos de iluminación convencionales en un primer costo, mientras que ofrecen un costo de propiedad significativamente menor (costo inicial más costo de electricidad) durante su ciclo de vida. El aumento de la eficacia y la reducción de los costos han permitido a los fabricantes de luminarias proporcionar un mejor rendimiento del color, distribución óptica, factor de forma y control avanzado de los productos de iluminación [32].

En la Tabla 1 se muestra una comparación entre las eficacias luminosas, y la vida útil de los LED de alto rendimiento y los productos de iluminación convencionales. Cuando la eficiencia de los productos LED se compara con los productos convencionales en cada categoría, se observa que son más eficaces que los productos de iluminación convencionales de mejor rendimiento. En conjunto, la mayor eficacia y la vida útil prolongada de las luminarias LED le brinda una ventaja competitiva en comparación con los productos de iluminación convencionales. Esto está estimulando su adopción, que a su vez resulta en ahorros significativos de energía.

TABLA 1. COMPARACIÓN DE LA EFICIENCIA DE LUMINARIAS CONVENCIONALES Y LOS SISTEMAS DE ILUMINACIÓN LED.

\begin{tabular}{|l|l|l|}
\hline Productores de led & Eficiencia de lluminación [Im/W] & Tiempo de vida útil [h] \\
\hline LED Lámpara A19 & 100 & 25.000 \\
Incandescente A19 & 15 & 1.000 \\
\hline LED Lámpara PAR38 & 88 & 25.000 \\
Alógena A19 & 20 & 8.400 \\
\hline LED tubo T8 & 149 & 50.000 \\
CFL Bombilla A19 & 70 & 12.000 \\
\hline LED de baja iluminación 6" & 86 & 50.000 \\
CFL Bombilla A19 dimerizable & 70 & 12.000 \\
\hline LED troffer 2"x4\$ & 129 & 50.000 \\
Sistema fluorescente lineal & 108 & 25.000 \\
\hline LED iluminación Vial & 118 & 60.000 \\
Sistemas HID (baja potencia) & 104 & 15.000 \\
\hline
\end{tabular}




\section{MARCO REGULATORIO DEL SECTOR DE LA ILUMINACIÓN EN COLOMBIA}

Para mejorar la eficiencia en la transformación de energía eléctrica, su uso y distribución, y con la gran necesidad de disminuir el impacto ambiental y el cambio climático generado por toda la cadena de energía eléctrica, el estado colombiano ha generado políticas públicas para el aumento de la eficiencia energética y el cuidado de los recursos naturales. Estas políticas se han implementado a través de diferentes estrategias tales como incentivos económicos, fiscales, regulación de precios, entre otros.

El estado ha fijado políticas públicas para aumentar la eficiencia energética, estas incluyen entre otras, la mejora y mantenimiento de la infraestructura del sistema eléctrico colombiano, la realización de investigaciones enfocadas a aumentar la eficiencia energética del país, la regulación de precios, impuestos y subsidios de acuerdo con cada sector de consumo energético, todo esto enmarcado en una política de protección de los recursos naturales. Las políticas públicas regulatorias desarrollan leyes y decretos de obligatorio cumplimiento cuya finalidad es proteger la vida humana, la vida animal y preservar el medio ambiente, de tal manera que se garantice la autonomía energética del país.

Las leyes y decretos son herramientas que cumplen la función de coaccionar a los actores del sector energético para evitar la adopción de comportamientos nocivos que puedan, de alguna manera, perjudicar el sistema energético del país, ya sea de manera económica, técnica o de algún otro tipo, so pena de multas, cierres parciales o totales de las entidades infractoras.

En Colombia, la Ley 697 de 2001 impulsa el uso racional y eficiente de la energía, estableciendo un marco jurídico para este propósito, y creando normas que establecen parámetros específicos en el desarrollo energético [34].

En un sistema de iluminación no toda la energía es aprovechada en iluminación, algún porcentaje de la energía se utiliza para energizar los circuitos necesarios para que las luminarias funcionen, otra parte de la energía se disipa en calor y alguna otra parte se convierte en contaminación de lumínica; esta contaminación es debida al reflejo de la luz, a brillo celestial, y al flujo incorrecto del haz de luz fuera de la luminaria.

Colombia cuenta con el Reglamento Técnico de Iluminación y Alumbrado Público (RETILAP), el cual presenta información técnica sobre el Uso Racional de la Energía (URE) e iluminación en general [35]. La tabla 2 presenta algunas regulaciones que el RETILAP considera como importantes para ser tenidas en consideración.

TABLA 2. PRINCIPALES RESOlUCIONES EN LA LEGISLACIÓN VIGENTE PARA LOS SISTEMAS DE ILUMINACIÓN EN COLOMBIA SEGÚN EL RETILAP [36].

\begin{tabular}{|c|c|}
\hline Resolución & eclaración \\
\hline $\begin{array}{l}181331 \text { de agosto } \\
6 \text { de } 2009\end{array}$ & igencia: se adopta el RETILAP que entraría en vigor el 20 de febrero de 2010. \\
\hline $\begin{array}{l}180265 \text { del } 19 \text { de } \\
\text { febrero de } 2010\end{array}$ & ntrada en vigor: se aplazó la entrada en vigor del reglamento hasta el $1^{\circ}$ de abril de 2010 . \\
\hline $\begin{array}{l}180540 \text { de marzo } \\
30 \text { de } 2010\end{array}$ & $\begin{array}{l}\text { equisitos de eficacia mínima y vida útil de fuentes lumínicas, además de otras disposiciones } \\
\text { ansitorias para facilitar su implementación. }\end{array}$ \\
\hline $\begin{array}{lll}181568 & \text { de } \\
\text { septiembre } & 1 & \text { de } \\
2010 & \end{array}$ & $\begin{array}{l}\text { Aclara y modificación al reglamento: en relación con el alcance a productos destinados a iluminación } \\
\text { decorativa y eficacias lumínicas de algunas fuentes. }\end{array}$ \\
\hline $\begin{array}{lll}182544 & \text { de } \\
\text { diciembre } & 29 & \text { de } \\
2010 & & \end{array}$ & $\begin{array}{l}\text { Ampliación de la transitoriedad: requisitos para bombillas incandescentes y la eficacia mínima para } \\
\text { tubos fluorescentes T8. }\end{array}$ \\
\hline $\begin{array}{l}180173 \text { de febrero } \\
14 \text { de } 2011\end{array}$ & htes. \\
\hline $\begin{array}{lll}91872 & \text { de } \\
\text { diciembre } 28 & \text { de } \\
2012 & & \end{array}$ & $\begin{array}{l}\text { Aclaracion en contenido de sustancias en las lumınarıas: maxımo contenıdo de mercurıo y plomo en } \\
\text { fuentes de iluminación y se aclaran algunos requisitos generales de balastos. }\end{array}$ \\
\hline $\begin{array}{ll}90980 & \text { de } \\
\text { noviembre } & 15 \text { de } \\
2013 & \end{array}$ & $\begin{array}{l}\text { Aspectos concernientes al Estatuto del Consumidor: se aclara y adiciona el RETILAP en relación a } \\
\text { incluir aspectos relevantes del Estatuto del Consumidor, así como de precisar los requisitos aplicables } \\
\text { a luminarias decorativas y de balastos. }\end{array}$ \\
\hline $\begin{array}{l}40122 \text { de febrero } 8 \\
\text { de } 2016\end{array}$ & $\begin{array}{l}\text { Adiciona y modifica en definiciones a las luminarias: aplicables a lámparas y luminarias que usan } \\
\text { tecnología LED, disponibilidad y acceso a información mínima de productos, extensión de plazo para } \\
\text { el uso de tecnología incandescente halógena y flexibilización de requisitos aplicables a luminarias } \\
\text { decorativas. }\end{array}$ \\
\hline
\end{tabular}




\section{CONCLUSIONES}

En este documento se presentó el resultado de una exploración de las tecnologías utilizadas para generar la iluminación artificial utilizada en diferentes tipos de ambientes, y se describe una clasificación de los sistemas de iluminación basada en la tecnología de la fabricación de la fuente emisora de luz; además, se hace recopilan datos de ciclo de vida y consumo de energía en algunas luminarias. Desde el punto de vista normativo, se hace una recopilación de información acerca de la legislación colombiana para el sector de iluminación, listando algunas leyes y decretos dispuestos por para su obligatorio cumplimiento, las cuales propenden por el cuidado de la vida humana, vegetal y animal, y en general de los equipos y dispositivos utilizados en los sistemas de energía. Además, en este documento se logró establecer las principales tecnologías en la construcción de luminarias en Colombia, y la tendencia a la modernización de los sistemas de iluminación, empleando tecnologías tales como luminarias de tipo T5 y LED.

En general, puede verse que el sector encargado de la iluminación está impulsando el desarrollo de tecnologías amigables que preserven el medio ambiente, diseñando dispositivos más eficientes, de tal manera que se pueda garantizar un sostenibilidad económica y ambiental. Entre los resultados a destacar de esta vigilancia, se logró identificar la cantidad de energía eléctrica que los sistemas de iluminación consumieron en varios períodos de la historia reciente en Colombia, y su tendencia de comportamiento en los próximos años; a partir de esta tendencia es posible decir que la cantidad de energía consumida en actividades de iluminación tiende a aumentar, debido a factores tales como el crecimiento de la población, a la diversidad de nuevas aplicaciones (decorativos, vigilancia, agricultura, y demás) de los sistemas de iluminación, y a la lentitud en el cambio de tecnologías.

\section{REFERENCIAS}

[1] O. D. Montoya Giraldo, A. Grajales, L. F. Grisales, and C. A. Castro, "Ubicación y Operación Eficiente de Almacenadores de Energía en Micro-redes en Presencia de Generación Distribuida," Rev. CINTEX, vol. 22, no. 1, pp. 97-117, Jun. 2017.

[2] E. A. Duque-Grisales, J. A. Patiño-Murillo, and L. D. Vélez-Gómez, "Aplicación del mercado de carbono en pequeñas centrales hidroeléctricas," Energética, vol. 44, pp. 19-32, 2014.

[3] J. Patino, C. A. Ramirez, and J. Espinosa, "Modal Analysis for a Power System Benchmark with Topological Changes," in Applied Computer Sciences in Engineering, vol. 1052, J. C. Figueroa-García, M. DuarteGonzález, S. Jaramillo-Isaza, A. D. Orjuela-Cañon, and Y. Díaz-Gutierrez, Eds. Cham: Springer International Publishing, 2019, pp. 628-639.

[4] S. Ruiz, J. Patiño, A. Marquez-Ruiz, J. Espinosa, E. Duque, and P. Ortiz, "Optimal Design of a Diesel-PVWind-Battery-Hydro Pumped POWER system with the Integration of ELECTRIC vehicles in a Colombian Community," Energies, vol. 12, no. 23, p. 4542, Nov. 2019, doi: 10.3390/en12234542.

[5] S. Ruiz Álvarez and J. J. Espinosa, "Control de potencia y velocidad de rotación de un aerogenerador usando controladores predictivos," Rev. CINTEX, vol. 23, no. 1, pp. 60-76, Oct. 2018, doi: 10.33131/24222208.311.

[6] J. Cardona Gil, J. H. Gallego Orrego, C. Isaza Roldán, R. Torres Salazar, and D. López Chejne, "Integración de Tecnologías Energéticamente Eficientes en Sistemas de Climatización Operados con Energía Térmica," Rev. CINTEX, vol. 22, no. 1, pp. 83-96, 2017.

[7] E. Duque Grisales and J. A. Patiño Murillo, "El mercado de bonos de carbono y su aplicación para proyectos hidroeléctricos," Rev. CINTEX, vol. 18, pp. 131-143, 2013.

[8] M. Fontoynont, "LED lighting, ultra-low-power lighting schemes for new lighting applications," Comptes Rendus Phys., vol. 19, no. 3, pp. 159-168, Mar. 2018, doi: 10.1016/j.crhy.2017.10.014.

[9] M. Stokes, M. Rylatt, and K. Lomas, "A simple model of domestic lighting demand," Energy Build., vol. 36, no. 2, pp. 103-116, Feb. 2004, doi: 10.1016/j.enbuild.2003.10.007.

[10] M. Cole, H. Clayton, and K. Martin, "Solid-State Lighting: The New Normal in Lighting," IEEE Trans. Ind. Appl., vol. 51, no. 1, pp. 109-119, Jan. 2015, doi: 10.1109/TIA.2014.2328790.

[11] F. Falchi et al., "The new world atlas of artificial night sky brightness," Sci. Adv., vol. 2, no. 6, p. e1600377, Jun. 2016, doi: 10.1126/sciadv.1600377.

[12] J. Bennie, J. Duffy, T. Davies, M. Correa-Cano, and K. Gaston, "Global Trends in Exposure to Light Pollution in Natural Terrestrial Ecosystems," Remote Sens., vol. 7, no. 3, pp. 2715-2730, Mar. 2015, doi: 10.3390/rs70302715.

[13] T. W. Davies, J. P. Duffy, J. Bennie, and K. J. Gaston, "The nature, extent, and ecological implications of marine light pollution," Front. Ecol. Environ., vol. 12, no. 6, pp. 347-355, Aug. 2014, doi: 10.1890/130281. 
[14] K. J. Gaston, J. P. Duffy, and J. Bennie, "Quantifying the erosion of natural darkness in the global protected area system: Decline of Darkness Within Protected Areas," Conserv. Biol., vol. 29, no. 4, pp. 1132 1141, Aug. 2015, doi: 10.1111/cobi.12462.

[15] T. W. Davies, J. P. Duffy, J. Bennie, and K. J. Gaston, "Stemming the Tide of Light Pollution Encroaching into Marine Protected Areas: Light pollution in marine protected areas," Conserv. Lett., vol. 9, no. 3, pp. 164171, May 2016, doi: 10.1111/conl.12191.

[16] N. B. Grimm et al., "Global Change and the Ecology of Cities," Science, vol. 319, no. 5864, pp. 756760, Feb. 2008, doi: 10.1126/science.1150195.

[17] T. Gallaway, R. N. Olsen, and D. M. Mitchell, "The economics of global light pollution," Ecol. Econ., vol. 69, no. 3, pp. 658-665, Jan. 2010, doi: 10.1016/j.ecolecon.2009.10.003.

[18] P. Morgan Pattison, M. Hansen, and J. Y. Tsao, "LED lighting efficacy: Status and directions," Comptes Rendus Phys., vol. 19, no. 3, pp. 134-145, Mar. 2018, doi: 10.1016/j.crhy.2017.10.013.

[19] C. Adarve Gómez, D. A. Castillo Carvajal, E. J. Restrepo Zapata, and H. Villar-Vega, "A review of virtual reality videogames for job-training applications," Rev. CINTEX, vol. 24, no. 1, pp. 64-70, Dec. 2019, doi: 10.33131/24222208.346.

[20] E. E. Dikel, G. R. Newsham, H. Xue, and J. J. Valdés, "Potential energy savings from high-resolution sensor controls for LED lighting," Energy Build., vol. 158, pp. 43-53, Jan. 2018, doi: 10.1016/j.enbuild.2017.09.048.

[21] T. G. Reames, M. A. Reiner, and M. B. Stacey, "An incandescent truth: Disparities in energy-efficient lighting availability and prices in an urban U.S. county," Appl. Energy, vol. 218, pp. 95-103, May 2018, doi: 10.1016/j.apenergy.2018.02.143.

[22] B. G. Bakshi and B. Roy, "Development \& simulation of dynamic conductance based high intensity discharge lamp model driven by low frequency square wave electronic ballast," in 2016 IEEE 7th Power India International Conference (PIICON), Bikaner, Rajasthan, India, 2016, pp. 1-6, doi: 10.1109/POWERI.2016.8077164.

[23] A. Rousseau and M. Guthrie, "Lightning Risk Assessment for Street Lighting Systems," in 2018 34th International Conference on Lightning Protection (ICLP), Rzeszow, 2018, pp. 1-6, doi: 10.1109/ICLP.2018.8503343.

[24] T. Babu and A. A. Balakrishnan, "Quadrupler DC converter for high intensity discharge lamp applications," in 2015 International Conference on Circuits, Power and Computing Technologies [ICCPCT2015], Nagercoil, India, 2015, pp. 1-6, doi: 10.1109/ICCPCT.2015.7159411.

[25] A. Farahat, A. Florea, J. L. Martinez Lastra, C. Branas, and F. J. Azcondo Sanchez, "Energy Efficiency Considerations for LED-Based Lighting of Multipurpose Outdoor Environments," IEEE J. Emerg. Sel. Top. Power Electron., vol. 3, no. 3, pp. 599-608, Sep. 2015, doi: 10.1109/JESTPE.2015.2453231.

[26] S. M. M. Rahman, J. Kim, G. Lerondel, Y. Bouzidi, and L. Clerget, "Value Retention Options in Circular Economy: Issues and Challenges of LED Lamp Preprocessing," Sustainability, vol. 11, no. 17, p. 4723, Aug. 2019, doi: 10.3390/su11174723.

[27] R. Lux, "TECNOLOGÍA LED," Renowatio LUX, 2019. [Online]. Available: http://renowatiolux.com/web/?page_id=8. [Accessed: 30-Sep-2019].

[28] C. Jiang, K. T. Chau, Y. Y. Leung, C. Liu, C. H. T. Lee, and W. Han, "Design and Analysis of Wireless Ballastless Fluorescent Lighting," IEEE Trans. Ind. Electron., vol. 66, no. 5, pp. 4065-4074, May 2019, doi: 10.1109/TIE.2017.2784345.

[29] Z. Y. Yu, V. K. Soo, and M. Doolan, "The Effect of Consumer Behaviour on the Life Cycle Assessment of Energy Efficient Lighting Technologies," Procedia CIRP, vol. 40, pp. 185-190, 2016, doi: 10.1016/j.procir.2016.01.097.

[30] M. F. da Silva et al., "Electric Equivalent Model for Induction Electrodeless Fluorescent Lamps," IEEE Trans. Power Electron., vol. 28, no. 7, pp. 3603-3613, Jul. 2013, doi: 10.1109/TPEL.2012.2227501.

[31] N. Gentile and M.-C. Dubois, "Field data and simulations to estimate the role of standby energy use of lighting control systems in individual offices," Energy Build., vol. 155, pp. 390-403, Nov. 2017, doi: 10.1016/j.enbuild.2017.09.028.

[32] N. Gentile, T. Laike, and M.-C. Dubois, "Lighting control systems in individual offices rooms at high latitude: Measurements of electricity savings and occupants' satisfaction," Sol. Energy, vol. 127, pp. 113-123, Apr. 2016, doi: 10.1016/j.solener.2015.12.053.

[33] UPME, "PLAN DE ACCIÓN INDICATIVO DE EFICIENCIA ENERGÉTICA 2017 - 2022," Ministerio de Minas y Energía, Plan de Acción, 2016.

[34] G. Mejía, "Estudio comparativo entre la legislación de eficiencia energética de Colombia y España," Rev. EAN, pp. 122-135, 2014. 
[35] A. Tafur Piedrahita et al., "Determinación de métricas de iluminación empleando metodología RETILAP, en ambiente de confecciones.," Rev. Teinnova, vol. 2, Jun. 2018, doi: 10.23850/25007211.1482.

[36] Ministerio de Minas y Energía, Reglamento Técnico de lluminación y Alumbrado Público - RETILAP. 2009 . 